\title{
Aktuelle Pränataldiagnostik: ein Überblick
}

\author{
Friderike Fornoff
}

\begin{abstract}
Neue Erkenntnisse im Bereich der medizinischen Forschung führen auch in der Pränataldiagnostik zu immer mehr Möglichkeiten. Mehr Wissen über das Ungeborene kann zur Gesundheit von Kind und Mutter beitragen. Aber nicht immer. Gelingt es nicht, das Wissen richtig zu deuten, so führt mehr Wissen zu mehr Unsicherheit. Dies kann eine Schwangerschaft nachhaltig belasten oder zu unnötigen Interventionen bei Mutter und Kind führen. Pränataldiagnostik ist daher sowohl fachlich als auch ethisch hoch komplex. Wer Paare in der Schwangerschaft betreut und berät, sollte die nachfolgend vorgestellten pränataldiagnostischen Verfahren kennen, einordnen und erklären können.
\end{abstract}

\section{Fragestellung vor der Untersuchung}

In diesem Artikel wird vor allem auf die Frage eingegangen, welche Möglichkeiten der Pränataldiagnostik aktuell zur Verfügung stehen und wie sie funktionieren.

Die weitaus schwierigere Frage, welche Untersuchungen durchgeführt werden sollten, kann hingegen immer nur individuell in Absprache mit der betroffenen Frau bzw. dem betroffenen Paar beantwortet werden.

Um dies zu ermöglichen, muss vor jeder Diagnostik sowohl dem/der ausführenden Arzt/Ärztin als auch dem Paar klar sein, was man mit der Diagnostik erkennen kann und was nicht.

Insbesondere über die Konsequenzen eines evtl. unerwünschten Testergebnisses für die Eltern und das Ungeborene sollte unbedingt vor der Testung gesprochen werden. Denn ist das Testergebnis da, lässt sich das Wissen darum nicht mehr rückgängig machen.

Hierfür braucht es interdisziplinäre fachliche Kompetenz und Einfühlungsvermögen, um den Ratsuchenden von möglichst vielen Seiten her zu einer autonomen Entscheidung zu verhelfen.

\section{Methoden}

Grundsätzlich werden invasive und nicht-invasive pränataldiagnostische Techniken unterschieden.

\section{Invasive Techniken}

Chorionzottenbiopsie (CVS = chorionic villous sampling)

Die CVS beschreibt eine Entnahme von Chorionzotten aus dem Plazentabett ( $\triangleright$ Abb.1). Die Punktion kann unter Ultraschallkontrolle entweder von abdominal oder von vaginal erfolgen.

Über die Frage ob ein vaginales Vorgehen mit einem höheren Abortrisiko einhergeht, herrscht Uneinigkeit unter Ärzten. Auch Studien zeigen hierzu widersprüchliche Ergebnisse [5][6][7][8]. Die meisten Pränataldiagnostiker wählen jedoch standardmäßig ein abdominales Vorgehen. Bei einer Hinterwandplazenta und oder bei retroflektiertem Uterus kann der vaginale Zugang allerdings eine Alternative darstellen, da die Plazenta hier häufig nur sehr schwierig oder gar nicht von abdominal zu erreichen ist.

Der Eingriff sollte nicht vor $11+0$ SSW erfolgen. Wird er davor durchgeführt, ist der Eingriff mit einem erhöhten Risiko für Extremitätenfehlbildungen der Feten assoziiert [3][4].

Die Chorionzottenbiopsie hat gegenüber der Fruchtwasserpunktion den Vorteil, dass sie deutlich früher durchgeführt werden kann und einen vorläufigen Befund des kompletten Chromosomensatzes schon nach 24 h ermöglicht.

Das Abortrisiko der CVS liegt ähnlich wie das der Fruchtwasserpunktion bei etwa 0,5-1\% [1][2]. 


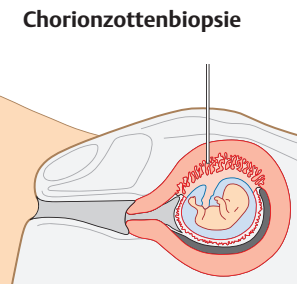

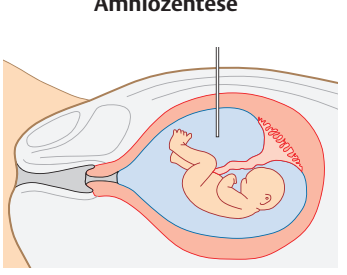

Chordozentese

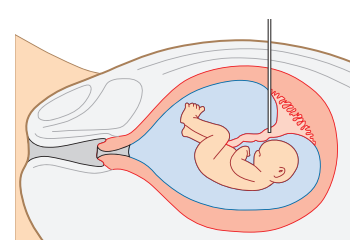

Abb. 1 bis 3: Chorionzottenbiopsie (links), Amniozentese (Mitte) und Chordozentese (rechts). (Quelle: Murken J, Kainer F. Untersuchungen vor Eintritt der Schwangerschaft. In: Murken J, Grimm T, Holinski-Feder E, et al., Hrsg. Taschenlehrbuch Humangenetik. 9. teilaktualisierte Auflage. Thieme; 2017. doi:10.1055/b-005-143305)

\section{Amniozentese (AC)}

Bei einer AC entnimmt man Fruchtwasser zu diagnostischen Zwecken. ( $\triangleright$ Abb. 2). Der Eingriff wird unter Ultraschallkontrolle und immer von abdominal durchgeführt. Es werden ca. $15 \mathrm{ml}$ Fruchtwasser entnommen.

Der Eingriff sollte erst ab 15 + 0 SSW durchgeführt werden, da bei Frühamniozentesen vor diesem Zeitraum ein deutlich erhöhtes Abortrisiko besteht und vermehrt Extremitätenfehlbildungen beschrieben wurden [9][10][11][12].

Zur Bestimmung des kompletten Karyotyps müssen erst Zellkulturen angelegt werden, sodass ein kompletter Karyotyp erst nach 10-14 Tagen vorliegt. Als Vorteil der AC können hier auch andere Parameter im Fruchtwasser gemessen werden (z. B. AFP = Alpha-feto-protein), sie ist zudem zur zusätzlichen Diagnostik bei Bauchwand und Neuralrohldefekten oder zum Virusnachweis bei V.a. Infektion geeignet.

Das Abortrisiko der AC liegt wie das der CVS bei etwa 0,5-1\% [1][2].

\section{Chordozentese (Nabelschnurpunktion)}

Hierbei wird fetales Blut aus der Nabelschnur entnommen ( Abb.3). Diese Technik wird insbesondere zur Anämiediagnostik genutzt, wenn im Ultraschall der Anhalt auf eine fetale Anämie besteht. Über diesen Zugang kann auch eine Bluttransfusion erfolgen. In der Regel wird eine Chordozentese nicht vor der 18+0 SSW durchgeführt. Die Komplikationsrate liegt mit 1,5-2\% deutlich höher als bei einer AC (13), sodass eine Chromosomenanalyse aus fetalem Blut nicht routinemäßig durchgeführt wird.

\section{GLOSSAR}

Detektionsrate: Die Detektionsrate (DR), oder Sensitivität eines Testverfahrens gibt an, wie viel Prozent der tatsächlich erkrankten Personen durch den Test auch als krank erkannt werden.
Falsch-positiv-Rate: Die FPR gibt an wie viel Prozent aller gesunden Personen durch den Test fälschlicherweise als krank erkannt werden.

\section{Nicht-invasive Techniken}

Bei den nicht-invasiven Techniken der Pränataldiagnostik soll hier insbesondere auf das Ersttrimesterscreening und die differenzialdiagnostische weiterführende Ultraschalluntersuchung des Feten im 2. Trimenon eingegangen werden.

\section{Erstes Screening}

Das in den Mutterschaftsrichtlinien vorgesehene erste Screening soll zwischen $8+0$ und $11+6$ SSW durchgeführt werden ( $\triangleright$ Abb. 7). Es umfasst lediglich die Feststellung, ob die Schwangerschaft intakt ist und intrauterin liegt. Des Weiteren sollen Mehrlingsgraviditäten und deren Chorionizität beschrieben sowie ggf. eine Terminkorrektur durch die Messung der SSL durchgeführt werden. Die Morphologie des Feten genauso wie eine Doppleruntersuchung wird nicht abgefragt. Wesentlich weiter gehen die Untersuchungen des Ersttrimesterscreenings (ETS), deren Kosten derzeit nicht von den gesetzlichen Krankenkassen übernommen werden.

\section{Ersttrimesterscreening (ETS)}

Beim ETS geht es um weit mehr als nur um die Messung der Nackenfalte. Diagnostische Fragestellungen des ETS können sein:

1. Wahrscheinlichkeitsberechnung für chromosomale Anomalien (Trisomie 21, 18, 13)

2. Ausschluss weiterer schwerwiegender Fehlbildungen (frühe Feindiagnostik)

3. Präeklampsie-Screening

Die Kosten belaufen sich je nach Praxis und Abrechnungsart auf ca. 120-300 Euro, die von den gesetzlichen Krankenassen derzeit nicht übernommen werden. 
- Tab. 1.1 Übersicht der Screening-Verfahren mit jeweiliger DR und FPR bezogen auf die Trisomie 21

\begin{tabular}{|l|l|l|l|l|l|}
\hline Screening-Verfahren & $\begin{array}{l}\text { mütterl. } \\
\text { Alter }\end{array}$ & Alter + NT & $\begin{array}{l}\text { Ersttrimester-Scree- } \\
\text { ning (Alter + NT+ } \\
\text { Biochemische } \\
\text { Parameter) }\end{array}$ & $\begin{array}{l}\text { Zweizeitiges } \\
\text { Ersttrimesterscreening }\end{array}$ & NIPT \\
\hline Detektionsrate (DR) & $50 \%$ & $80 \%$ & $90 \%$ & $95 \%$ & $99 \%$ \\
\hline Falsch-Positivrate (FPR) & $25 \%$ & $5 \%$ & $5 \%$ & $3 \%$ & $0,04 \%$ \\
\hline
\end{tabular}

\section{Wahrscheinlichkeitsberechnung für chromosomale Anomalien (Trisomie 21, 18, 13)}

Schon immer gab es Kinder, die mit Chromosomenstörungen auf die Welt kamen. Von jeher bestand, zumindest für Teile der Gesellschaft, das Bedürfnis ein solches Ereignis besser vorhersehen zu können. Ende der 1960er Jahre war es erstmals möglich, schon vor der Geburt fetale Zellen durch eine Fruchtwasseruntersuchung zu gewinnen, auf ihren Karyotypen untersuchen zu lassen und somit eine Chromosomenstörung beim ungeborenen Kind vorhersagen zu können.

Eine routinemäßige AC bei allen Schwangeren empfand man jedoch schon damals für nicht gerechtfertigt und zu risikoreich. Das Ziel sollte aber sein, soviel Chromosomenstörungen wie möglich mit so wenig invasiven Untersuchungen wie nötig zu erkennen.

\section{Risikofaktor Alter}

Das Risiko ein Kind mit einer Chromosomenstörung zu gebären nimmt mit steigendem Alter der Mutter zu (20). Mit 35 Jahren ist die Wahrscheinlichkeit, einen pathologischen Befund zu erhalten erstmals so hoch wie das Fehlgeburtsrisiko der Amniocentese.

Aus diesem Grund entschied man sich, nur Frauen ab 35 Jahren eine AC anzubieten.

Auch heute noch zahlen die gesetzlichen Krankenkassen allen Frauen über 35 Jahren, die es wünschen, eine Amniocentese oder eine CVS. Ärzte sind dazu verpflichtet, die Frauen über diese Möglichkeit aufzuklären.

Auf diese Weise könnte man heute ca. 50\% der Schwangerschaften mit Chromosomenstörungen entdecken. Die anderen $50 \%$ der Kinder mit Chromosomenstörungen werden von Frauen geboren, die unter 35 Jahre alt sind.

Zwar ist bei jeder jüngeren Frau das Risiko geringer ein Kind mit Chromosomenstörungen zu gebären, aber es gibt viel mehr schwangere Frauen unter 35 als über 35 Jahren. So kommt es, dass Kinder mit Chromosomenstörungen zu gleichen Teilen von Frauen unter und über 35 Jahren geboren werden.
Verwenden wir nur das Alter der Patientin als nicht-invasiven Screening-Test und würde allen Frauen über 35 Jahren eine AC empfohlen, so hätte dieser Test heute eine Entdeckungsrate (Detektionsrate $=D R$ ) von nur etwa $50 \%$. Allerdings läge die Falsch-Positiv-Rate (FPR) bei ca. $25 \%$. Das bedeutet, dass bei fast einem Viertel der gesunden Kinder der Test fälschlicherweise positiv ausfallen würde.

Diese DR gepaart mit der FPR ist für einen Screeningtest unzureichend. Daher versuchten Pränataldiagnostiker weitere Screeningparameter als das Alter der Schwangeren zu finden.

\section{Nackenfalte / Nackentransparenz (NT)}

Bei der Nackentransparenz handelt es sich um eine physiologische Ansammlung von Flüssigkeit unter der fetalen Haut im Bereich der Halswirbelsäule ( $\triangleright$ Abb 4).

Einen Zusammenhang zwischen einer verdickten NT und fetalen Chromosomenanomalien konnte erstmals in den 1980er Jahren gezeigt werden. Die Londoner Arbeitsgruppe um Professor Nicolaides entwickelte bald darauf einen Screening-Algorithmus, in den sowohl das Alter der Schwangeren als auch die sonographisch gemessene Breite der Nackenfalte in der $11+0-13+6$ SSW (bzw. Scheitel-Steiß-Länge (SSL) 45-84 mm) eingingen.

Auf alle Schwangeren angewendet, erreicht man für die Trisomie 21, 18 u. 13 so eine DR von ca. $80 \%$ bei einer FPR von nur 5 \% [21]. Dem Ziel, so viele Chromosomenstörungen wie möglich mit so wenigen ACs wie nötig zu entdecken, war man so ein großes Stück nähergekommen.

Um einen verlässlichen Wert einer NT für diese Risikoberechnung zu erhalten, müssen bestimmte Kriterien erfüllt werden. Die Untersuchung ist nicht trivial und bedarf einiger Erfahrung in der Sonographie. Um eine möglichst hohe Qualität der NT-Messungen zu gewährleisten, haben sich in Deutschland zwei Zertifizierungsverfahren durchgesetzt, bei denen Untersucher an einem Kurs teilnehmen und regelmäßig von ihnen gemessene Bilder einreichen müssen. 


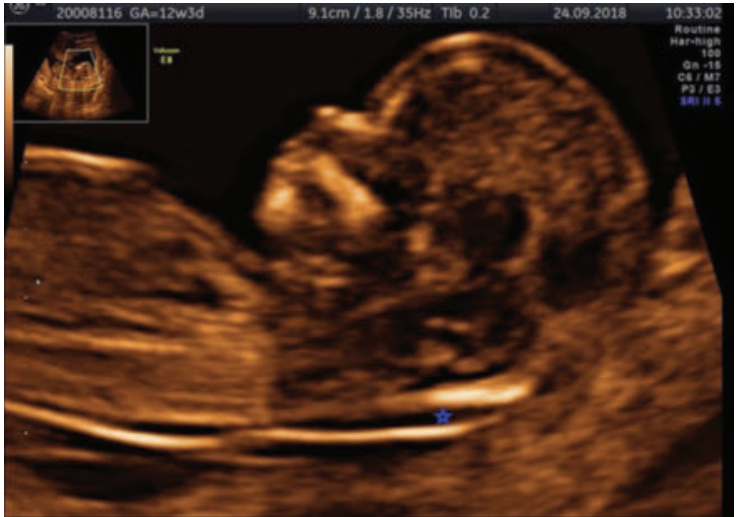

-Abb. 4 Die Nackentransparenzmessung ermittelt die physiologische Ansammlung von Flüssigkeit unter der fetalen Haut im Bereich der Halswirbelsäule (siehe blaue Markierung). (Quelle: Friderike Fornoff)

In Deutschland sind dies die FMF (Fetal Medicine Foundation)-Deutschland und die FMF-London. Die hier angegebenen DR und FPR beziehen sich auf die FMF-London.

\section{Biochemische Parameter PAPP-A und B-HCG}

Auch die Konzentrationen bestimmter während der Schwangerschaft im Blut nachweisbarer Hormone sind bei Feten mit Chromosomenstörungen verändert.

Sie unterscheiden sich unterschiedlich stark je nach Schwangerschaftswoche und Art der Chromosomenstörung. Für den Zeitraum des ETS eignen sich hier besonders PAPP-A und B-HCG.

Bezieht man die Konzentration dieser Hormone im mütterlichen Blut zusätzlich zur Nackenfaltenmessung im Zeitraum $11+0-13+6$ SSW in die Risikobewertung mit ein, so kann die DR (bei gleicher FPR) von $80 \%$ auf $90 \%$ gesteigert werden [22].

\section{Zweizeitiges Screening}

Wird die Blutentnahme in der 10. SSW und die NT-Messung in der 13. SSW durchgeführt, das ETS also zu zwei Zeitpunkten durchgeführt, kann eine weitere Verbesserung der DR auf etwa $95 \%$ und der FPR auf $3 \%$ erreicht werden [23]. Das führt dazu, dass - die qualitativ hochwertige NT-Messung vorausgesetzt - weniger Frauen unnötig verunsichert, aber mehr Chromosomenstörungen erkannt werden.

\section{Zusätzliche Ultraschallmarker}

Auch weitere Marker können in die Risikoberechnung einfließen und zu einer weiteren Verbesserung der DR und einer Verringerung der FPR führen.

Ein häufig verwendeter Marker, ist das fetale Nasenbein. Ist es zur Zeit des ETS sonographisch noch nicht darstellbar, steigt die Wahrscheinlichkeit für eine Chromosomenstörung deutlich an.
Weitere Marker sind der Nachweis einer undichten rechten Herzklappe (Trikuspidalklappeninsuffizienz) oder eines Rückflusses im Ductus venosus, was indirekt ein Hinweis auf einen Herzfehler und somit auch für eine Chromosomenstörung sein kann.

\section{Testergebnis des ETS}

Das Ergebnis des ETS wird als Wahrscheinlichkeit für die Chromosomenstörungen Trisomie 21, 13 und 18 angegeben. Es wird unterschieden zwischen hohem, mittlerem und niedrigem Risiko. Die Cut-off-Werte für ein niedriges Risiko sind je nach Fachgesellschaft unterschiedlich. Bei der FMF-London gilt ein Risiko von $<1: 1000$ als niedrig. Liegt das Risiko unter 1:1000, wird keine weitere Diagnostik veranlasst.

Der Cut-off-Wert für ein hohes Risiko wird kontrovers diskutiert und hat sich durch neuere Untersuchungsmethoden wie den NIPT (s. u. ) eher hin zu größeren Risiken entwickelt. Er wird zumeist bei> 1:50 - 1:10 angenommen. Besteht ein höheres Risiko als 1:10 sollte eine invasive Diagnostik durchgeführt werden. Aufgrund der frühen Woche, in der das ETS durchgeführt wird, erfolgt die invasive Diagnostik meist mittels CVS.

Alle Risiken zwischen 1:10 und 1:1000 gelten als mittleres Risiko. Über Jahre entsprach dies einer diagnostischen Grauzone in der das Risiko für eine Chromosomenstörung zwar nicht als niedrig gelten konnte, aber auch nicht als hoch genug, um eine invasive Diagnostik zu rechtfertigen. Dieses Dilemma des mittleren Risikos lässt sich seit 2012 durch den sogenannten Nicht invasiven Pränataltest (NIPT) auflösen.

\section{Früher Fehlbildungsausschluss}

Nimmt man alle schwerwiegenden fetalen Fehlbildungen zusammen, machen die Chromosomenstörungen nur etwa $8 \%$ der Fehlbildungen aus. Der weitaus größere Teil (ca. 92\%) betrifft chromosomal unauffällige Kinder. Verzichtet man auf die detaillierte Ultraschalluntersuchung und ersetzt sie durch einen Nicht invasiven Pränataltest (NIPT, s. Box), entdeckt man zwar mit einer sehr hohen Wahrscheinlichkeit ein Kind mit einer Trisomie 21, die mit einer vergleichsweise milden Form der geistigen Behinderung einhergeht. Man übersieht aber möglicherweise andere wesentlich schwerwiegendere Fehlbildungen, die im Ultraschall erkennbar wären.

In einer Untersuchung von Syngelaki et al. konnte 2011 gezeigt werden, dass zum Zeitpunkt des ETS sonographisch $31 \%$ aller fetalen Fehlbildungen immer und immerhin $43 \%$ manchmal dargestellt werden können [16].

In der Detektionsrate aller schwerwiegenden Fehlbildungen zusammen ist der Ultraschall dem NIPT daher immer noch überlegen. 


\section{STICHWORT}

\section{Nicht invasiver Pränataltest (NIPT)}

Der Nicht invasive Pränataltest oder zellfreie DNA-Test (Non-invasive prenatal Testing = NIPT) beschreibt eine neuere Methode zum Screening auf Chromosomenstörungen, die jedoch nicht zum ETS im engeren Sinne zählt.

Während der Schwangerschaft befinden sich neben der mütterlichen auch die kindliche Erbinformation im Blut der Mutter. Diese liegen allerdings nicht als vollständige Chromosomen, sondern in Form von zellfreien DNA-Bruchstücken vor. Die kindliche zellfreie DNA stammt vom kindlichen Anteil der Plazenta. In den späten 1990er Jahren gelang erstmals der Nachweis von freier fetaler DNA im Blutplasma schwangerer Frauen [14].

Seit 2012 sind in Deutschland kommerzielle Tests auf dem Markt, die freie fetale DNA im mütterlichen Blut mit unterschiedlichen Methoden untersuchen und bestimmte Chromosomenstörung beim Kind mit hoher Wahrscheinlichkeit vorhersagen können. Die DR für die Trisomien 21, 18 und 13 liegen bei > $99 \%, 98 \%$ und 99,0\%, einer Gesamt- FPR von $0,13 \%$ und sind den zuvor beschriebenen Methoden des ETS damit deutlich überlegen [24].

Aber auch ein auffälliger NIPT ist lediglich eine Screeninguntersuchung und bedarf immer einer invasiven Diagnostik zur endgültigen Diagnosesicherung. Sollte die invasive Diagnostik einen normalen Chromosomensatz zeigen, so ist das Kind chromosomal gesund.

Leider gibt es immer wieder Fälle, in denen Eltern, möglicherweise aufgrund des guten Marketings der Firmen, den NIPT-Ergebnissen mehr Glauben schenken als einer unauffälligen invasiven Diagnostik. Wir können leider auch von Fällen berichten, in denen Eltern sich innerhalb der Fristenregelung für einen Schwangerschaftsabbruch bei chromosomal gesunden Kindern aufgrund eines vorher auffälligen NIPTs entschlossen haben.

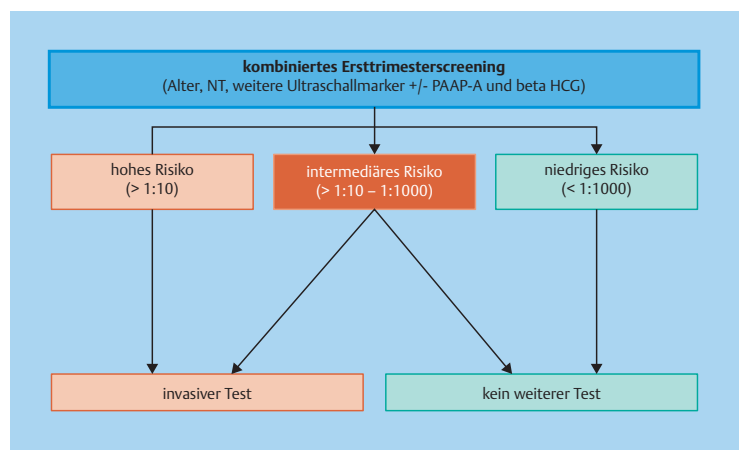

Abb. 5 Algorithmus Ersttrimester-Screening.
Derzeit ist der NIPT als Screeningverfahren ab der 10 + 0 SSW für folgende chromosomale Auffälligkeiten verfügbar: Trisomie 21, 13, 18 und geschlechtschromosomale Anomalien. Seit Kurzem besteht auch die Möglichkeit, auf die Mirkodeletion 22q11 zu screenen (Di-George-Syndrom), der PanoramaTest $^{\mathrm{TM}}$ bietet zusätzlich ein Screening auf weitere Mikrodeletions-Syndrome und Triploidien an. Diese Untersuchungen sind bisher jedoch noch nicht weitläufig etabliert und werden von vielen Fachleuten nicht als Routineuntersuchung empfohlen.

Noch vor einigen Jahren kostete eine solche Untersuchung über 1000 Euro. Heute liegen die Kosten je nach Fragestellung bei etwa 250 bis 450 Euro.

Die Tests sind u. a. unter den Handelsnamen Praenatest ${ }^{\mathrm{TM}}$, Harmony-Test ${ }^{\mathrm{TM}}$ und Panorama-Test ${ }^{\mathrm{TM}}$ bekannt. Der NIPT wird bisher nicht von den gesetzlichen Kassen übernommen. Bei auffälligen ETS kann jedoch eine Kostenübernahmeerklärung erfragt werden. Eine grundsätzliche Kostenübernahme durch die gesetzlichen Krankenkassen wird diskutiert. Nicht immer erhalten wir bei einem NIPT ein Ergebnis. Insbesondere bei übergewichtigen Patientinnen oder in frühen Schwangerschaftswochen ist der Anteil der fetalen freien DNA im mütterlichen Blut häufig zu gering, um sie auszuwerten.

Bezogen auf das ETS bietet der NIPT im Falle eines mittleren Risikos (s. o. ) eine sehr gute Ergänzung. Daher sollte allen Schwangeren mit einem mittleren Risiko im ETS ein NIPT empfohlen werden. Ist dieser unauffällig, können die Chromosomenstörungen Trisomie 13, 18 und 21 mit sehr hoher Wahrscheinlichkeit ausgeschlossen werden und es kann auf eine invasive Diagnostik verzichtet werden ( $\triangleright$ Abb. 5 und Abb. 6).

Warum man das aufwendige ETS nicht durch den einfacheren NIPT ersetzt, erklärt sich daraus, dass der NIPT den Ausschluss von schwerwiegenden Fehlbildungen nicht leisten kann, einen ebenfalls wichtigen Bestandteil des ETS.

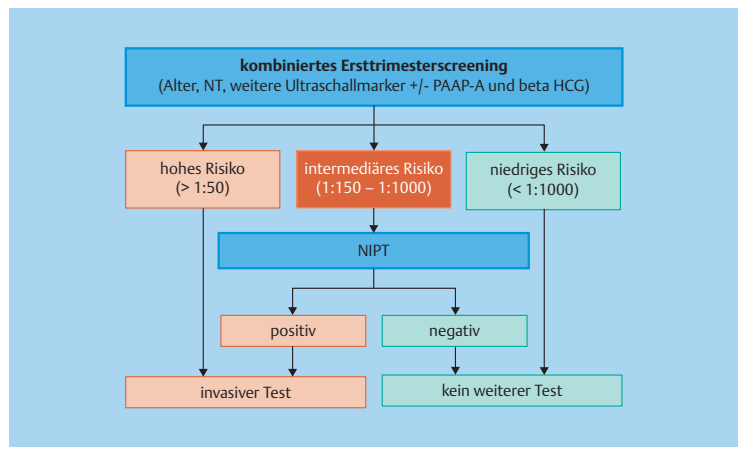

Abb. 6 Kombination aus Ersttrimester-Screening und Nicht-invasivem Pränataltest. 


\section{Präeklampsie-Screening}

Eine weitere wichtige Möglichkeit im Rahmen des ETS ist das Risikoscreening für die Entwicklung einer Präeklampsie und einer frühen fetalen Wachstumsretardierung (IUGR) im weiteren Schwangerschaftsverlauf. Analog zur Risikoberechnung für die Chromosomenstörungen wird hier mithilfe anamnestischer (Alter der Mutter, geburtshilfliche Vorgeschichte, Parität, BMI, vorbestehende Erkrankungen und familiäre Belastungen mit Präeklampsie), sonographischer (Dopplermessung der Aa. uterinae bds), klinischer (Blutdruckmessungen bds.) und laborchemischer Parameter (PAPP-A und ggf. PIGF) eine Risikoberechnung erstellt. Auf diese Weise lassen sich 95\% der Schwangerschaften identifizieren, die später unbehandelt aufgrund einer Präeklampsie oder IUGR in eine Geburt vor der 34. SSW resultieren würden [17].

Unterschiedliche Studien konnten zeigen, dass die Verabreichung von 100-150 mg ASS täglich, begonnen vor der 16 SSW, das Risiko, in der späteren Schwangerschaft eine Präeklampsie oder IUGR zu entwickeln, um bis zu zwei Drittel senken kann [18][19].

Die FPR des Präeklampsie-Screenings liegt bei $10 \%$. Kritiker führen diese relativ hohe FPR als Argument gegen ein flächenhaftes frühes Screening auf Präeklampsie ins Feld. Derzeit wird ein Screening auf Präeklampsie im Rahmen des ETS hauptsächlich in pränatalmedizinischen Abteilungen und Praxen durchgeführt. Zumindest Frauen mit anamnestischen Risiken für Präeklampsie sollten jedoch über die Möglichkeit des Screenings aufgeklärt werden.

\section{Zweites Screening}

Selbstverständlich kann man während der gesamten Schwangerschaft sonographische Feindiagnostik durchführen. Der optimale Zeitpunkt, zu dem die Anatomie weit genug entwickelt und das Kind groß genug ist, um auch komplizierte Strukturen wie das Herz ausreichend gut zu beurteilen, liegt in der $20+0$ bis $22+0$ SSW. Ist die Schwangerschaft weiter fortgeschritten wird die Beurteilung aufgrund der Größe des Kindes wieder schwieriger.

Etwa zu dieser Zeit ist auch in den Mutterschaftsrichtlinien ein Ultraschallscreening vorgesehen $(18+0-21+6$ SSW $)$.

Im Wesentlichen sind 3 Untersuchungen von sehr unterschiedlichem Umfang möglich.

Aufgrund der Tatsache, dass die Begriffe für die jeweiligen Untersuchungsumfänge nicht immer gleich verwendet werden, kommt es hier häufig zu Begriffsverwirrungen.

\section{Basisultraschall}

Die einfachste Form ist der Basisultraschall. Hierbei werden im Wesentlichen die Größe des Kindes, der Plazentasitz und die Fruchtwassermenge beurteilt. Der Untersuchungsinhalt ist im Mutterpass auf Seite 10 und 11 dunkelgrau hinterlegt ( Abb.7). Die Untersuchung kann in Deutschland von jedem Frauenarzt durchgeführt werden und wird von den gesetzlichen Kassen übernommen.

\section{Erweiterter Basisultraschall}

Seit 2013 muss allen Schwangeren unabhängig von Alter und Schwangerschaftsrisiken der sog. erweiterte Basisultraschall angeboten werden. Hierbei handelt es sich um eine etwas differenziertere Beurteilung der kindlichen Anatomie. Es werden u. a. Bauchwand und Rücken beurteilt sowie die Lage und das Aussehen des Herzens und einige Strukturen des kindlichen Köpfchens. Die untersuchten Strukturen sind ebenfalls im Mutterpass auf Seite $10 \mathrm{u} 11 \mathrm{zu}$ finden und sind dort hellgrau hinterlegt ( $>$ Abb. 7). Der erweiterte Basisultraschall ist immer eine Kassenleistung.

Der erweiterte Basisultraschall kann von allen Frauenärzten durchgeführt werden, die sich über eine Online-Prüfung dafür qualifiziert haben. Besondere praktische Erfahrungen in der Sonographie müssen nicht nachgewiesen werden. Durch diese Neuerung erhofft man sich ein besseres Screening durch die niedergelassenen Frauenärzte, um so eine höhere präpartale Erkennungsrate von schweren Fehlbildungen zu erreichen.

\section{Differenzialdiagnostische weiterführende Ultraschalluntersuchung}

Vom erweiterten Basisultraschall zu unterscheiden ist die differenzialdiagnostische weiterführende Ultraschalluntersuchung des Feten. Hierbei handelt es sich um einen erweiterten Fehlbildungsausschluss, der im Vergleich zum erweiterten Basisultraschall wesentlich umfangreicher ist. Aufgrund des sperrigen Begriffs werden hier häufig die Bezeichnungen Organscreening, Fehlbildungsausschluss, Feindiagnostik o. ä. verwendet. Dies kann für den Laien oft verwirrend sein, da diese oder ähnliche Begriffe auch für den erweiterten Basisultraschall verwendet werden.

Die Durchführung der differenzialdiagnostischen weiterführenden Ultraschalluntersuchung des Feten und deren Interpretation sind sehr anspruchsvoll und bedürfen einiger Erfahrung. Daher sollte er ausschließlich von Ärztinnen bzw. Ärzten mit einer entsprechenden Qualifikation und ausreichender Kenntnis der Pränataldiagnostik erbracht werden. Um die differenzialdiagnostische weiterführende Ultraschalluntersuchung des Feten mit den gesetzlichen Krankenkassen abrechnen zu können, bedarf es einer besonderen Genehmigung durch die Kassenärztliche Vereinigung. Die Deutsche Gesellschaft für Ultraschall in der Medizin (DEGUM) sieht für diese Untersuchung die Qualifikation des Untersuchers nach DEGUM Stufe II oder III vor, während die Voraussetzung 
für den erweiterten Basisultraschall in etwa der DEGUM Stufe I entspricht.

\begin{abstract}
Merke
Beim erweiterten Basisscreening und der differenzialdiagnostischen weiterführenden Ultraschalluntersuchung des Feten liegt der Schwerpunkt darauf, Fehlbildungen der Kinder zu erkennen, die entweder zum Schutze des Kindes eine Änderung des geburtshilflichen Managements verlangen oder einer intensivierten kinderärztlichen Versorgung direkt postpartal bedürfen. Das Wissen um solche Fehlbildungen schon präpartal und die damit mögliche vorzeitige Planung des perinatalen Procederes, z. B. Entbindung in einer Klinik mit angegliederter Kinderklinik, Kinderchirurgie und / oder sogar Kinderkardiochirurgie, kann so zu einer wesentlichen Prognoseverbesserung des Kindes führen.
\end{abstract}

In bestimmten Fällen kann es für die Eltern auch bei weniger schweren Fehlbildungen helfen, sich mental auf die Fehlbildung vorzubereiten. Ein gutes Beispiel hierfür ist die Lippen-Kiefer-Gaumen-Spalte.

Hier können die Konfrontation mit Bildern vor und nach Therapie deutlich den Schrecken abmildern, den die Eltern haben mögen, wenn sie ihr Kind mit einer Spaltbildung nach der Geburt das erste Mal sehen. Auch die erschwerte Stillperiode kann in diesem Fall besser vorbereitet werden.

\title{
Indikationen für Feindiagnostik
}

Von den gesetzlichen Kassen wird die differenzialdiagnostische weiterführende Ultraschalluntersuchung des Feten für ausgewählte Indikationen übernommen:

- Verzicht auf Amniozentese bei Schwangeren ab 35 Jahren

- Nichtdarstellbarkeit oder Auffälligkeit im BasisUltraschallscreening

- Geschwisterkinder oder Eltern mit Fehlbildungen (bes. Herzfehler) oder Chromosomenanomalien

- mütterliche Erkrankungen mit erhöhtem Fehlbildungsrisiko ( z. B. Diabetes, Adipositas)

- Verdacht fetaler Infektionen (z. B. Toxoplasmose, CMV)

- Medikamenteneinnahme mit potenzieller Teratogenität

- monochoriale Mehrlingsschwangerschaft

- Verwandtenehe

- auffälliges ETS

- psychosoziale Indikation (Fehlbildungsangst)

Ohne Indikation ist die differenzialdiagnostische weiterführende Ultraschalluntersuchung eine Individuelle Gesundheitsleistung (IGEL), deren Kosten ca. 200-300 Euro liegen und von den Eltern selbst getragen werden müssen. 


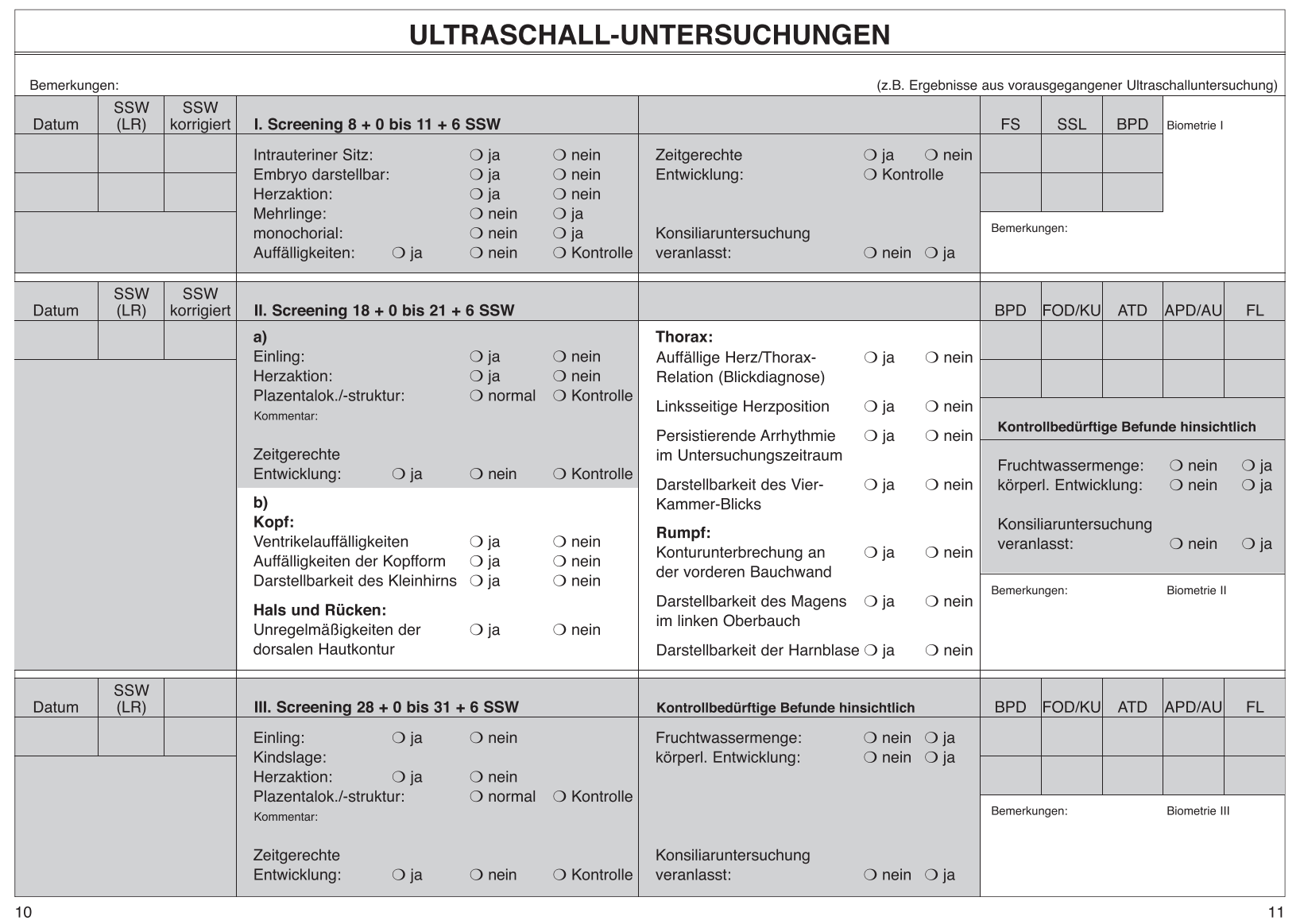

Abb. 7 Ultraschall-Untersuchungen im Mutterpass. Quelle: Gemeinsamer Bundesausschuss (G-BA), juristische Person des öffentlichen Rechts, Wegelystr. 8, 10623 Berlin.

\section{Weitere Möglichkeiten der Pränataldiagnostik}

Auf zusätzliche Möglichkeiten der Pränataldiagnostik soll hier nicht weiter eingegangen werden, da sie nicht Gegenstand der gewöhnlichen Vorsorge sind. Hierzu zählen u.a. das fetale MRT, welches zunehmend an Bedeutung gewinnt. Insbesondere beim Verdacht auf neurologische Fehlbildungen kann ein fetales MRT oft genaueren Aufschluss über die vermutete Fehlbildung geben. Wichtig ist hierbei, dass wie bei jeder Bildgebung sichergestellt sein sollte, dass die untersuchende Institution über ausreichend Erfahrung in der Beurteilung fetaler MRTs verfügt.

\section{FAZIT}

\section{Take Home Messages}

- Über die Konsequenzen eines evtl. unerwünschten Testergebnisses für die Eltern und das Ungeborene sollte unbedingt vor der Testung gesprochen werden.

- Als invasive Testverfahren stehen v. a. die Fruchtwasseruntersuchung und die Chorionzottenbiopsie zur Verfügung. Wobei die CVS schon früher (ab der $11+0$ SSW) durchführbar ist.

- Das Ersttrimesterscreening ist mehr als nur „die Nackenfalte messen“. Neben der Wahrscheinlichkeitsberechnung einer Chromosomenstörung kann es eine frühe Fehlbildungsdiagnostik und ein Screening auf Präeklampsie beinhalten

- Als Screeningtest für die Trisomie 21, 13 und 18 ist der NIPT dem ETS überlegen. Zur endgültigen Diagnosestellung bedarf es aber auch hier unbedingt der invasiven Diagnostik.

- Mehr als $90 \%$ aller angeborenen schweren Fehlbildungen kommen bei Kindern ohne Chromosomenstörung vor. Diese erkennt der NIPT nicht. Sie können aber im Rahmen eines ETS erkannt werden.

- Die differenzialdiagnostische weiterführende Ultraschalluntersuchung des Feten unterscheidet sich deutlich vom erweiterten Basisscreening und sollte nur von in der sonographischen Pränataldiagnostik erfahrenen Ärztinnen und Ärzten durchgeführt werden. 


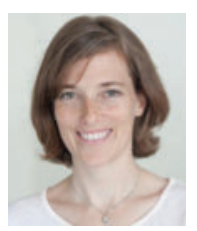

Dr. med. Friderike Fornoff ist Ärztin für Frauenheilkunde, spezielle Geburtshilfe und Perinatalmedizin, Pränataldiagnostik Degum II in Frankfurt am Main.

\section{Korrespondenzadresse}

Praxis Am Salzhaus

Dr. med. Friderike Fornoff

Kaiserstraße 6

60311 Frankfurt am Main

E-Mail: F.Fornoff@gynprenat.de

\section{Literatur}

[1] Alfirevic Z, Navaratnam K, Mujezinovic F. Amniocentesis and chorionic villus sampling for prenatal diagnosis. Cochrane Database Syst Rev. 2017 Sep 4;9:CD003252.

[2] Mujezinovic F, Alfirevic Z. Procedure-related complications of amniocentesis and chorionic villous sampling: a systematic review. Obstet Gynecol. 2007 Sep;110(3):687-94. Review. Erratum in: Obstet Gynecol. 2008 Mar;111(3):779

[3] Mastroiacovo P, Botto LD, Cavalcanti DP, Lalatta F, Selicorni A, Tozzi AE, Baronciani D, Cigolotti AC, Giordano S, Petroni F, et al. Limb anomalies following chorionic villus sampling: a registry based case-control study. Am J Med Genet. 1992 Dec 1;44(6):856-64. Review.

[4] Dolk H, Bertrand F, Lechat MF. Chorionic villus sampling and limb abnormalities. The EUROCAT Working Group.Lancet. 1992 Apr 4;339(8797):876-7.

[5] Jackson LG, Zachary JM, Fowler SE, Desnick RJ, Golbus MS, Ledbetter DH, Mahoney MJ, Pergament E, Simpson JL, Black $\mathrm{S}$, et al. A randomized comparison of transcervical and transabdominal chorionic-villus sampling. The U.S. National Institute of Child Health and Human Development ChorionicVillus Sampling and Amniocentesis Study Group. N Engl J Med. 1992 Aug 27;327(9):594-8.

[6] Brambati B, Terzian E, Tognoni G. Randomized clinical trial of transabdominal versus transcervical chorionic villus sampling methods. Prenat Diagn. 1991 May;11(5):285-93.

[7] Silver RK, MacGregor SN, Muhlbach LH, Kambich MP, Ragin A. A comparison of pregnancy loss between transcervical and transabdominal chorionic villus sampling. Obstet Gynecol. 1994 May; 83 (5 Pt 1): 657-60.

[8] Chueh JT, Goldberg JD, Wohlferd MM, Golbus MS. Comparison of transcervical and transabdominal chorionic villus sampling loss rates in nine thousand cases from a single center. Am J Obstet Gynecol. 1995 Oct;173(4):1277-82.

[9] Canadian Early and Mid-trimester Amniocentesis Trial (CEMAT) Group. Randomised trial to assess safety and fetal outcome of early and midtrimester amniocentesis. Lancet 1998; 351: $242-247$

[10] Nicolaides K, Brizot Mde L, Patel F et al. Comparison of chorionic villus sampling and amniocentesis for fetal karyotyping at 10 - 13 weeks' ge- station. Lancet 1994; 344: 435 - 439

[11] Tabor A, Alfirevic Z. Update on Procedure-Related Risks for Prenatal Di- agnosis Techniques. Fetal Diagn Ther 2010; 27 : 1 - 7
[12] Royal College of Obstetricians and Gynaecologists. Amniocentesis and Chorion Villous Sampling, Green-top Guideline No 8; 2010

[13] Tongsong T, Wanapirak C, Kunavikatikul C, Sirirchotiyakul S, Piyamongkol W, Chanprapaph P. Fetal loss rate associated with cordocentesis at midgestation. Am J Obstet Gynecol. $2001 \mathrm{Mar} ; 184(4): 719-23$.

[14] Lo YMD, Corbetta N et. al. Presence of fetal DNA in maternal plasma and serum. Lancet 1997; 350: 485-7.

[15] Lo YMD, Chan, KCA, et.al. Maternal plasma DNA sequencing reveals the genom- wide genetic and mutational profile of the fetus. Sci Transl Med 2010; 2:61ra91

[16] Syngelaki A, Chelemen T, Dagklis T, Allan L, Nicolaides KH. Challenges in the diagnosis of fetal non-chromosomal abnormalities at 11-13 weeks. Prenat Diagn.2011 Jan;31(1):90-102. doi: 10.1002 / pd.2642.

[17] Poon LC, Syngelaki A, Akolekar R, Lai J, Nicolaides KH. Combined screening for preeclampsia and small for gestational age at 11-13 weeks. Fetal Diagn Ther. 2013;33(1):16-27. doi: 10.1159/000341712. Epub 2012 Sep 13.

[18] Bujold E, Roberge S, Lacasse Y, Bureau M, Audibert F, Marcoux $S$, Forest JC, Giguère Y. Prevention of preeclampsia and intrauterine growth restriction with aspirin started in early pregnancy: a meta-analysis. Obstet Gynecol. 2010 Aug; 116 (2 Pt 1): 402-14.

[19] Rolnik DL, Wright D, Poon LC, O'Gorman N, Syngelaki A, de Paco Matallana C, Akolekar R, Cicero S, Janga D, Singh M, Molina FS, Persico N, Jani JC, Plasencia W, Papaioannou G, TenenbaumGavish K, Meiri H, Gizurarson S, Maclagan K, Nicolaides KH. Aspirin versus Placebo in Pregnancies at High Risk for Preterm Preeclampsia. N Engl J Med. 2017 Aug 17;377(7):613-622. doi: 10.1056 / NEJMoa1704559. Epub 2017 Jun 28.

[20] Hook EB. Rates of chromosome abnormalities at different maternal ages. Obstet Gynecol. 1981 Sep;58(3):282-5.

[21] Snijders RJ, Noble P, Sebire N, Souka A, Nicolaides KH. UK multicentre project on assessment of risk of trisomy 21 by maternal age and fetal nuchal-translucency thickness at 10-14 weeks of gestation. Fetal Medicine Foundation First Trimester Screening Group. Lancet. 1998 Aug 1;352 (9125):343-6.

[22] Nicolaides KH, Spencer K, Avgidou K, Faiola S, Falcon O. Multicenter study of first-trimester screening for trisomy 21 in 75 821 pregnancies: results and estimation of the potential impact of individual risk-orientated two-stage first-trimester screening. Ultrasound Obstet Gynecol. 2005 Mar;25(3):221-6.

[23] Kagan KO, Wright D, Baker A, Sahota D, Nicolaides KH. Screening for trisomy 21 by maternal age, fetal nuchal translucency thickness, free beta-human chorionic gonadotropin and pregnancy-associated plasma protein-A. Ultrasound Obstet Gynecol. 2008 Jun;31(6):618-24.

[24] Gil MM, Accurti V, Santacruz B, Plana MN, Nicolaides KH. Analysis of cell-free DNA in maternal blood in screening for aneuploidies: updated meta-analysis. Ultrasound Obstet Gynecol. 2017 Sep;50(3):302-314.

\section{Bibliografie}

DOI https://doi.org/10.1055/a-0729-5280

Die Hebamme 2018; 31: 304-313

(c) Georg Thieme Verlag KG Stuttgart · New York ISSN 0932-8122 